\title{
Free 25-Hydroxyvitamin D Levels Are Normal in Subjects with Liver Disease and Reduced Total 25-Hydroxyvitamin D Levels
}

Daniel D. Bikle, * Bernard P. Halloran," Elaine Gee," Elizabeth Ryzen, $\neq$ and John G. Haddad\$

*Veterans Administration Medical Center and Department of Medicine, University of California, San Francisco, California 94121 ; $\ddagger$ Department of Medicine, University of Southern California, Los Angeles, California 90033; §Department of Medicine, University of Pennsylvania, Philadelphia, Pennsylvania 19104

\begin{abstract}
We determined the free fraction of 25-dihydroxyvitamin $D$ (250HD) in the serum of subjects with clinical evidence of liver disease and correlated these measurements to the levels of vitamin D binding protein and albumin. These subjects when compared to normal individuals had lower total 250HD levels, higher percent free $250 \mathrm{HD}$ levels, but equivalent free $250 \mathrm{HD}$ levels. These subjects also had reduced vitamin $\mathrm{D}$ binding protein and albumin concentrations. The total concentration of $250 \mathrm{HD}$ correlated positively with both vitamin $D$ binding protein and albumin, whereas the percent free $250 H D$ correlated negatively with vitamin $D$ binding protein and albumin. The free $250 \mathrm{HD}$ levels did not correlate with either vitamin $D$ binding protein or albumin. We conclude that total vitamin D metabolite measurements may be misleading in the evaluation of the vitamin $D$ status of patients with liver disease, and recommend that free 250HD levels also be determined before making a diagnosis of vitamin $\mathbf{D}$ deficiency.
\end{abstract}

\section{Introduction}

Patients with liver disease often present with low circulating levels of 25-hydroxyvitamin D (25OHD) ${ }^{1}(1-3)$. This metabolite of vitamin $D$ is produced by the liver (4), and its measurement is used to assess the vitamin D status of the patient. The correlation of 25OHD levels with various indices of liver function (1) suggests that patients with liver disease have a compromised capacity to metabolize vitamin D to $250 \mathrm{HD}$. Since patients with liver disease frequently have bone disease in association with low 25OHD levels $(5,6)$, various forms of vitamin $D$ have been used as therapy for such patients (7). However, osteoporosis, not osteomalacia (as would be expected in vitamin D-deficient states), is the predominant form of bone disease in most patients with cirrhosis (8-12), and in most studies $(5,9-10)$ neither vitamin $D$ nor 25OHD therapy has been effective in treating this type of osteoporosis. Furthermore, 250HD levels do not correlate well with the extent of osteomalacia (6), and a deficiency in 25OHD production by the cirrhotic liver has not been conclusively demonstrated $(2,11,13)$.

\footnotetext{
Address reprint requests to Dr. Bikle.

Received for publication 13 January 1986 and in revised form 13 May 1986.
}

1. Abbreviations used in this paper: $1,25(\mathrm{OH})_{2} \mathrm{D}, 1,25$-dihydroxyvitamin D; DBP, vitamin D binding protein; 25OHD, 25-hydroxyvitamin D; HPLC, high performance liquid chromatography.

The Journal of Clinical Investigation, Inc.

Volume 78, September 1986, 748-752
One explanation for low levels of the vitamin D metabolites in patients with liver disease without osteomalacia is that the carriers of the vitamin D metabolites in serum, vitamin $D$ binding protein (DBP) and albumin (14-17), are also low. DBP and albumin are produced in the liver (18), and the levels of both are reduced in the serum of patients with liver disease (19). A reduction of DBP and albumin concentrations would reduce the total concentration of the vitamin $\mathrm{D}$ metabolites without reducing the free or unbound concentrations. Therefore, if the free vitamin $\mathrm{D}$ metabolite concentrations are the physiologically important concentrations with respect to target tissue response, then total vitamin D metabolite measurements may not accurately indicate the vitamin $\mathrm{D}$ status of patients with liver disease.

We recently (20) adapted the technique of centrifugal ultrafiltration (21) to measure free 1,25-dihydroxyvitamin D $\left(1,25[\mathrm{OH}]_{2} \mathrm{D}\right)$ in serum and observed that patients with liver disease have normal free $1,25(\mathrm{OH})_{2} \mathrm{D}$ levels despite low total $1,25(\mathrm{OH})_{2} \mathrm{D}$ levels. In this report, we applied this method to the measurement of free 250HD levels. Our results indicate that patients with liver disease had low total $250 \mathrm{HD}$ levels that correlated with their low DBP and albumin levels, but most of these patients had normal free 25OHD levels.

\section{Methods}

Subjects. Blood was obtained from 25 normal subjects aged 24-50 yr ( 13 males, 12 females) and 42 subjects aged 22-70 yr (34 males, 8 females) with clinical evidence of liver disease. Three of the normal females were using oral contraceptives. Nearly all of the subjects with liver disease had a history of alcohol abuse; cirrhosis was documented by biopsy in 19. Two of the subjects with liver disease were taking diphenylhydantoin, and one had been given oral supplements of vitamin D before the blood sample was obtained. With these exceptions, the subjects were not taking drugs known to affect the vitamin D endocrine system. Excluding these subjects from the data analysis did not alter the conclusions. A history of fractures associated with trauma was discovered in two of the subjects with liver disease.

Analyses. The free fraction of $250 \mathrm{HD}$ in serum for all of the subjects was determined using the following modification of centrifugal ultrafiltration originally developed by Hammond et al. (21). $450 \mu \mathrm{l}$ of serum, diluted $1 / 100$ with $0.01 \mathrm{M}$ phosphate-buffered saline $\mathrm{pH} 7.4$ (PBS), was incubated at $37^{\circ} \mathrm{C}$ for $45 \mathrm{~min}$ with $0.1 \mu \mathrm{Ci}\left[{ }^{3} \mathrm{H}\right] 25 \mathrm{OHD} \mathrm{H}_{3}(150-160 \mathrm{Ci} /$ mmol) (Amersham Corp., Arlington Heights, IL) and 7,500 dpm $\left[{ }^{14} \mathrm{C}\right]$ glucose, both of which had been dried under $\mathrm{N}_{2}$ before addition of the serum. The $\left[{ }^{3} \mathrm{H}\right] 25 \mathrm{OHD} \mathrm{H}_{3}$ was purified the day of the assay using a Waters high performance liquid chromatography (HPLC) system (Waters Associates, Milford, MA) equipped with a Zorbax Sil column (E. I. Dupont, Wilmington, DE) and a solvent system of 97:3 hexane/isopropanol. The serum sample was then divided into two $200-\mu$ l aliquots that were each placed into the inner vial of the centrifugal ultrafiltration apparatus. (This vial consisted of a 11/4-in. section of a $12 \times 75 \mathrm{~mm}$ borosilicate tube closed at one end with dialysis tubing [A. H. Thomas, Philadelphia, PA] with a molecular weight cut-off of 12,000 .) A 10- $\mu$ l aliquot was obtained to verify complete solubilization of the tracers. The inner vial 
rested on three No. 1 filter pads (Whatman, Hillsboro, OR) within a scintillation vial, and both were centrifuged at $800 \mathrm{~g}$ for $90 \mathrm{~min}$ in a centrifuge at $37^{\circ} \mathrm{C} .30 \mu \mathrm{l}$ of the contents of the inner vial (the serum sample) and the entire contents of the outer vial (the ultrafiltrate) were analyzed for ${ }^{3} \mathrm{H}$ and ${ }^{14} \mathrm{C}$ by liquid scintillation spectroscopy. By this method, the ultrafiltrate at $90 \mathrm{~min}$ measured $\sim 30 \mu$ l. The calculation of the free fraction (F) of $250 \mathrm{HD}$ is as follows: $F=\left[{ }^{3} \mathrm{H}\right] \mathrm{u} /\left[{ }^{14} \mathrm{C}\right] \mathrm{u}$ $\div\left[{ }^{3} \mathrm{H}\right] \mathrm{s} /\left[{ }^{14} \mathrm{C}\right] \mathrm{s}$, where $\mathrm{u}=$ the ultrafiltrate and $\mathrm{s}=$ the serum. Free $25 \mathrm{OHD}$ levels were calculated as the product of the free fraction and total $250 \mathrm{HD}$ level.

Equilibrium dialysis to measure the free fraction of $250 \mathrm{HD}$ was performed in Plexiglas units with chambers separated by the same dialysis tubing used for the centrifugal ultrafiltration vials. In this method, 1.6 $\mathrm{ml}$ of the diluted serum was incubated with $0.2 \mu \mathrm{Ci}$ freshly purified $\left[{ }^{3} \mathrm{H}\right] 25 \mathrm{OHD}_{3}$, and two $0.75-\mathrm{ml}$ aliquots were dialyzed each against 2.6 $\mathrm{ml}$ PBS for up to $96 \mathrm{~h}$ at $37^{\circ} \mathrm{C}$. At the end of dialysis, aliquots from each side of the chamber were analyzed for ${ }^{3} \mathrm{H}$ and the free fraction of 25OHD was calculated as: $\left[{ }^{3} \mathrm{H}\right] \mathrm{b} /\left[{ }^{3} \mathrm{H}\right] \mathrm{s}$, where $\mathrm{b}=$ the buffer side and $\mathrm{s}$ $=$ the serum sample side. After removal of the original buffer and sample, the units were washed with methanol to extract any $\left[{ }^{3} \mathrm{H}\right] 25 \mathrm{OHD}$ that adhered to the sides.

The free fraction of $250 \mathrm{HD}$ was also estimated using a commercially available ultrafiltration device (Centricon, Amicon Corp., Danvers, MA). The membrane of the unit was washed sequentially with $0.1 \mathrm{M} \mathrm{NaOH}$, $70 \%$ ethanol, $10 \mathrm{mM} \mathrm{NaHCO}, 2 \mathrm{mM}$ EDTA, water, and PBS in a fashion similar to the washing of the dialysis membranes used for equilibrium dialysis and centrifugal ultrafiltration described above. Serum samples were prepared as for centrifugal ultrafiltration; $0.5-\mathrm{ml}$ aliquots were added to the upper chamber of the Centricon apparatus and the samples were then centrifuged at $800 \mathrm{~g}$ for $10 \mathrm{~min}$ at $37^{\circ} \mathrm{C}$. The entire ultrafiltrate $(\sim 50 \mu \mathrm{l})$ and a $30-\mu \mathrm{l}$ aliquot of the sample were analyzed for ${ }^{3} \mathrm{H}$ and ${ }^{14} \mathrm{C}$; the free fraction was calculated as for the results from centrifugal ultrafiltration described above.

Total 25OHD levels in serum were determined in 54 of the subjects by the method of Eisman et al. (22) using chemically synthesized $25 \mathrm{OHD}_{3}$ (a gift from J. D. Babcock, Upjohn Co., Kalamazoo, MI) as standard. DBP levels were measured in 52 of the subjects by rocket immunoelectrophoresis (23) using purified human DBP as standard and monospecific antiserum developed in rabbits. Albumin levels in 64 of the subjects, calcium levels in 51 of the subjects, phosphorus levels in 42 of the subjects, and creatinine levels in 38 of the subjects were measured by automated clinical laboratory techniques.

Statistical analyses used the statistical package SAS and employed these procedures: correlation, Student's $t$ test, plot, and general linear models (for linear regression).

\section{Results}

In initial experiments, we compared the methods of centrifugal ultrafiltration and equilibrium dialysis. Equilibrium dialysis for $48 \mathrm{~h}$ resulted in free $250 \mathrm{HD}$ levels that were one-half to onethird that obtained by centrifugal ultrafiltration. However, we observed that when the free metabolite was dialyzed into the buffer solution across the membrane of the dialysis chamber, much of the metabolite adhered to the sides of the chamber and equilibrium was not achieved even after $96 \mathrm{~h}$. Furthermore, the free metabolite decomposed during this time, and only a small fraction of the tracer was recovered as $250 \mathrm{HD}$ from the buffer side of the dialysis chamber. In contrast, $85-100 \%$ of the tracer in the ultrafiltrate from centrifugal ultrafiltration was recovered as $250 H D$ when rechromatographed with the HPLC system used in its purification.

We then compared results from our method of centrifugal ultrafiltration using two vials to those from the use of a commercially available ultrafiltration device. The free fraction of $25 \mathrm{OHD}$ obtained using the commercially available device was one-third to one-tenth that obtained using the two-vial method. However, most of the 25OHD that passed through the membrane of the commercial device bound to the plastic support underneath the membrane and was not recovered in the ultrafiltrate. The two-vial centrifugal ultrafiltration apparatus that we used to obtain the data for this report is constructed such that the dialysis membrane covering the one end of the inner vial rests directly on filter pads in the bottom of the outer collection vial. During at least 120 min of centrifugation, $\left[{ }^{3} \mathrm{H}\right] 25 \mathrm{OHD}$ appeared in the collection vial in parallel with the $\left[{ }^{14} \mathrm{C}\right]$ glucose used to mark the free water of the ultrafiltrate, indicating that the $\left[{ }^{3} \mathrm{H}\right] 25 \mathrm{OHD}$ is not being preferentially absorbed by the filter pads during the centrifugation. Therefore, we conclude that centrifugal ultrafiltration using the two-vial method accurately measured the free fraction of $250 \mathrm{HD}$, whereas equilibrium dialysis and ultrafiltration using the commercially available apparatus underestimated this measurement.

The very low free fraction of $250 \mathrm{HD}$ in serum $(0.00030 \pm 0.00007$ in normal individuals) prevents an accurate assessment in whole serum; therefore, the serum was diluted before the assay was performed. Dilutions of serum from 1/30 to $1 / 300$ with PBS gave equivalent results when corrected to whole serum. At higher dilutions (1/1000 and above), the high affinity binding site for 25OHD in serum (DBP) began to be saturated with the concentration of $\left[{ }^{3} \mathrm{H}\right] 25 \mathrm{OHD}$ in the sample $(\sim 1.5 \mathrm{nM})$, and at such dilutions the apparent free 25OHD level was no longer linear with the dilution of the serum.

The $\mathrm{pH}$ of serum adjusted with 0,5 , or $10 \% \mathrm{CO}_{2}$ did not affect the measured free $250 \mathrm{OH}$ level. Varying the incubation time of 25OHD in serum from 5 to $60 \mathrm{~min}$ before centrifugation did not affect the measured free 250HD level. Increasing the duration of centrifugation from 30 to 120 min did not affect the measured free $250 H D$ level. In six consecutive assays, the intraand intercoefficients of variation were 9.5 and $8 \%$, respectively.

The serum levels of calcium, phosphorus, and creatinine in normal subjects and in subjects with liver disease are shown in Table I. The serum calcium levels were lower in the subjects with liver disease $(9.7 \pm 0.4$ vs. $8.1 \pm 0.7 \mathrm{md} / \mathrm{dl}, P<0.001)$. However, as Table II shows, the albumin levels in the subjects with liver disease were also reduced $(4.46 \pm 0.24$ vs. $2.83 \pm 0.66 \mathrm{~g} / \mathrm{dl})$. Serum calcium was highly correlated with serum albumin levels ( $r=0.899, P<0.001)$. Serum phosphorus and creatinine levels did not differ significantly between the two groups of subjects.

The serum levels of total 25OHD, percent free 25OHD, free 25OHD, DBP, and albumin in normal subjects and subjects with liver disease are shown in Table II. In the serum of subjects with liver disease, the DBP was $44 \%$ of normal, the albumin was $59 \%$ of normal, and the total $250 H D$ was $57 \%$ of normal. The percentage of the total 25OHD that was free in the subjects with liver disease was 0.068 or $226 \%$ of normal. The free concentra-

Table I. Serum Levels of Calcium, Phosphorus, and Creatinine in Normal Subjects and Subjects with Liver Disease

\begin{tabular}{llll}
\hline & Calcium & Phosphorus & Creatinine \\
\hline & $m g / d l$ & $m g / d l$ & $m g / d l$ \\
Liver disease \pm SD & $8.1 \pm 0.7^{*}$ & $3.2 \pm 1.0$ & $1.1 \pm 0.5$ \\
Normals \pm SD & $9.7 \pm 0.4$ & $3.4 \pm 0.5$ & $0.8 \pm 0.2$
\end{tabular}

$* P<0.001$ compared to normal. 
Table II. Serum Levels of Total and Free 25OHD, DBP, and Albumin (Alb)

in the Serum of Subjects with Liver Disease and Normal Controls

\begin{tabular}{llllll}
\hline Group & DBP & Alb & Total 250HD & Percent free 250HD & Free 250HD \\
\hline & $\mu g / m l$ & $g / d l$ & $n g / m l$ & $p g / m l$ \\
Liver disease \pm SD & $178 \pm 92^{*}$ & $2.83 \pm 0.66^{*}$ & $10.9 \pm 9.5 \ddagger$ & $0.068 \pm 0.029^{*}$ & $6.61 \pm 4.61$ \\
Normal \pm SD & $405 \pm 128$ & $4.46 \pm 0.24$ & $19.2 \pm 6.7$ & $0.030 \pm 0.007$ & $5.88 \pm 2.27$ \\
\hline
\end{tabular}

* $P<0.0001$ compared to normal. $\ddagger P<0.0005$ compared to normal.

tions of $250 \mathrm{OHD}(6.61 \pm 4.6 \mathrm{pg} / \mathrm{ml})$ in these subjects were not significantly different from normal $(5.88 \pm 2.27 \mathrm{pg} / \mathrm{ml})$.

The total 25OHD was positively correlated to DBP ( $r$ $=0.571, P<0.0001)$ and albumin $(r=0.562, P<0.001)$ (Fig. 1) when data from both cirrhotic and normal subjects were combined. The correlations remained significant $(r=0.465, P$ $<0.02 ; r=0.367, P<0.05$ for the correlations of 25OHD to DBP and albumin, respectively) when data from the cirrhotic subjects were analyzed separately, but not when data from normal individuals were analyzed separately. The percent free 250HD was negatively correlated to $\operatorname{DBP}(r=0.766, P<0.0001)$ and albumin $(r=0.586, P<0.0001)$ when data from both cirrhotic and normal subjects were combined, although the plot of percent free 25OHD and DBP was not linear (Fig. 2). At a DBP concentration above $300 \mu \mathrm{g} / \mathrm{ml}$, little further reduction in percent free $250 H D$ was observed. The correlation of percent free 25OHD to DBP remained significant when data from cirrhotic subjects were analyzed separately $(r=0.693, P<0.0001)$; this correlation was not significant for the data from normal subjects, and the correlation for percent free 25OHD to albumin was not significant for data from either group analyzed separately. The free 25OHD did not correlate to either DBP or albumin
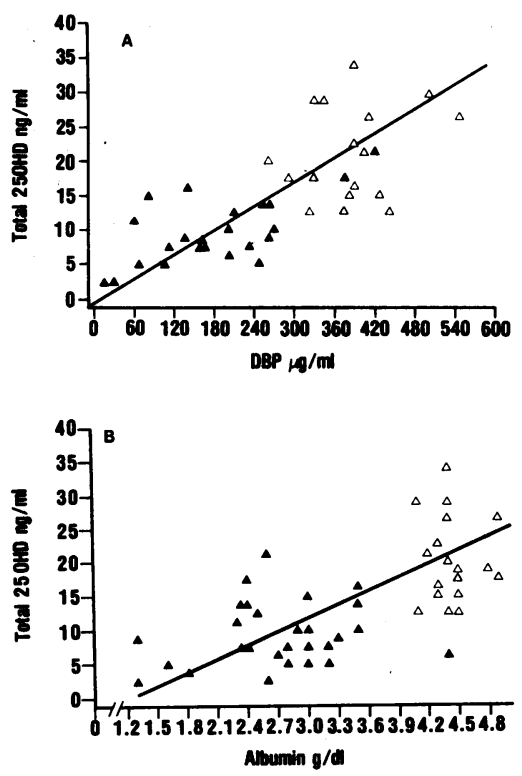

Figure 1. The correlation of the level of total 25-hydroxyvitamin D with the level of DBP $(A)$ in 24 subjects with liver disease $(\Delta)$ and in 17 normal subjects $(\Delta)$, and with the level of albumin $(B)$ in 29 subjects with liver disease $(\Delta)$ and in 18 normal subjects $(\Delta)$. Only data from samples in which the correlated measurements were made in the same sample are included. whether the data from both groups of subjects were combined or separated (Fig. 3). Neither the free 25OHD nor the total 250HD correlated to serum calcium, phosphorus, or creatinine levels when the influence of albumin was factored out by multiple analysis of variance.

\section{Discussion}

The serum level of $250 H D$ is often measured as an index of vitamin D status. Reduced 25OHD levels are observed in vitamin $\mathrm{D}$ deficiency and a number of gastrointestinal and hepatic dis-
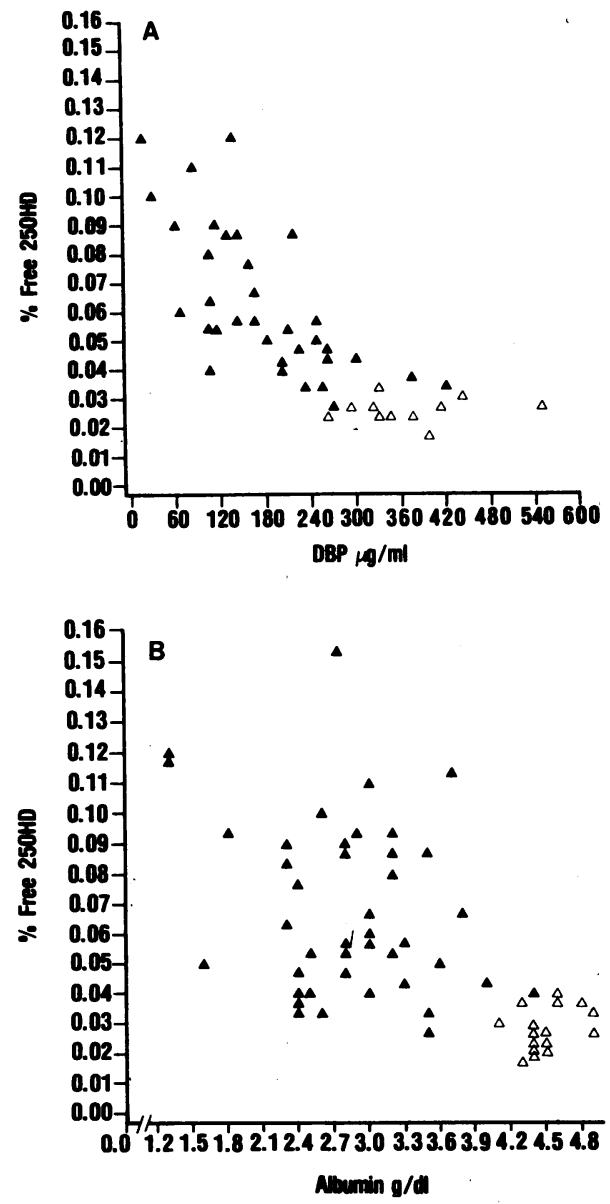

Figure 2. The correlation of the level of percent free 25-hydroxyvitamin D with the level of DBP levels $(A)$ in 34 subjects with liver disease ( $\Delta)$ and in 11 normal individuals $(\Delta)$, and with the level of albu$\min (B)$ in 42 subjects with liver disease $(\Delta)$ and in 16 normal subjects $(\Delta)$. Only data from samples in which the correlated measurements were made in the same sample are included. 

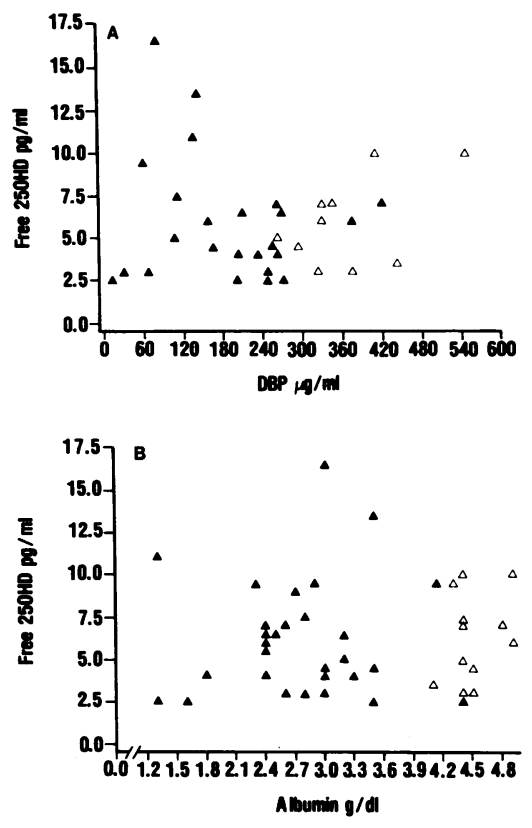

Figure 3. The correlation of the level of free 25-hydroxyvitamin D with both the level of DBP $(A)$ in 24 subjects with liver disease $(\Delta)$ and in 10 normal subjects $(\Delta)$, and the level of albumin $(B)$ in 29 subjects with liver disease $(\Delta)$ and in 12 normal subjects $(\Delta)$. Only data from samples in which both measurements were made in the same sample are included.

eases (24). Because 25OHD is produced by the liver, the low 25OHD levels associated with liver disease have been attributed to reduced production of $250 \mathrm{HD}$ by the diseased liver (25). A number of studies have failed to demonstrate a significant loss of $250 H D$ production in patients with liver disease $(2,11,13)$, however, and the correlation between the serum levels of 25OHD and osteomalacia in patients with liver disease is poor (7). In this study, we found that the free concentration of 250HD in subjects with liver disease was normal, despite the low total concentration of $250 \mathrm{OHD}$. This result is consistent with our previous finding (20) that the free $1,25(\mathrm{OH})_{2} \mathrm{D}$ level was also normal in a similar group of subjects. We did not evaluate these subjects for the presence of bone disease, but in several recent reports $(11,26)$ of similar patients (alcoholics) in whom bone disease was evaluated, osteoporosis, not osteomalacia, was observed. Osteoporosis in association with liver disease has been difficult to treat with vitamin $D(5,7,8,12)$, indicating that vitamin $D$ deficiency may not be a major cause of the osteoporosis.

The correlation of total 250HD and percent free 25OHD to both DBP and albumin reflects not only the fact that DBP, albumin, and 25OHD are produced in the liver, but that DBP and albumin both participate in the transport of the vitamin $D$ metabolites in blood. We (17) recently have reported that in normal individuals, $\sim 85 \%$ of the $1,25(\mathrm{OH})_{2} \mathrm{D}$ in blood is bound to DBP and nearly $15 \%$ is bound to albumin. In subjects with liver disease and lower DBP concentrations, a higher percentage of the $1,25(\mathrm{OH})_{2} \mathrm{D}$ is bound to albumin. Using a similar approach, we (27) have calculated that $88 \%$ of the serum $250 \mathrm{HD}$ was bound to DBP in normal subjects, whereas this fell to $83 \%$ in patients with liver disease.

The assessment of free 25OHD in serum is complicated by the small percentage of free $250 \mathrm{OD}$ in serum $(0.03 \%)$, its poor solubility in aqueous media, its tendency to adhere to plastic and glassware, and its instability when exposed to light, heat, and oxygen. Accordingly, we used large amounts $(0.1 \mu \mathrm{Ci})$ of freshly purified $\left[{ }^{3} \mathrm{H}\right] 25 \mathrm{OHD}$ for each assay and employed diluted $(1 / 100)$ serum. These conditions enabled complete solubilization of the tracer, while providing a readily measurable fraction of free metabolite. When the two-vial apparatus was used, the entire contents of the ultrafiltrate were absorbed by filter pads in the collection vial. If the $\left[{ }^{3} \mathrm{H}\right] 25 \mathrm{OHD}$ were selectively absorbed by the filter pads in comparison to the $\left[{ }^{14} \mathrm{C}\right]$ glucose, this technique would overestimate the free fraction of $250 \mathrm{HD}$. However, the $\left[{ }^{14} \mathrm{C}\right]$ glucose and $\left[{ }^{3} \mathrm{H}\right] 25 \mathrm{OHD}$ appeared in the ultrafiltrate at the same ratio throughout centrifugation, indicating that selective absorption did not occur under the conditions of our assay. Other techniques that we have used to measure free 250HD have not proved satisfactory because the $\left[{ }^{3} \mathrm{H}\right] 25 \mathrm{OHD}$ decomposed during the long incubation period required for equilibrium dialysis and adhered to the filter support of commercially available ultrafiltration devices.

The amount of $\left[{ }^{3} \mathrm{H}\right] 25 \mathrm{OHD}$ added to the serum samples (diluted 1/100) is of the same order of magnitude as the endogenous $25 \mathrm{OHD}\left(\sim 10^{-9} \mathrm{M}\right)$. However, because of the excess of DBP and albumin even in the diluted samples $\left(\sim 5 \times 10^{-8} \mathrm{M}\right.$ and $\sim 7 \times 10^{-6} \mathrm{M}$, respectively), the additional amount of 250HD does not alter the fraction of $250 \mathrm{HD}$ bound to these proteins. In other experiments, we have not observed a change in the free fraction of $250 \mathrm{HD}$ until concentrations of $250 \mathrm{HD}$ in the sample approached those of the DBP concentrations.

This study reports the first directly measured free 250HD concentrations in human serum. Only $0.03 \%$ of total $250 H D$ in serum was free in normal individuals. This percentage was increased in subjects with liver disease who had reduced DBP and albumin levels, a finding that is consistent with the role of DBP and albumin in transporting the vitamin D metabolites in blood. Because of the large excess capacity of DBP and albumin for $250 \mathrm{OH}$, in the absence of a change in affinity of these proteins for $250 H D$, the percent free $250 H D$ is reciprocally related to the DBP and albumin concentrations by the equilibrium constant of the binding reaction. The rise in percent free 25OHD in the serum of subjects with liver disease compensated for the fall in total $250 H D$ levels such that the free $250 H D$ levels remained normal in most patients. This observation may account for part of the difficulty in correlating total 25OHD levels to the presence of osteomalacia in subjects with liver disease. We believe that measurement of free $250 \mathrm{HD}$ in subjects with liver disease will provide important additional information in assessing their vitamin D status.

\section{Acknowledgments}

We acknowledge the help with statistical analysis by Dr. Judy Evaul of the Walter Reed Army Medical Center, the editorial assistance of Cheryl Holzaepfel, and the graphics assistance of Irene Bertolucci.

This work was supported by grants from the Veterans Administration and the National Institutes of Health (AM 28116).

\section{References}

1. Hepner, G. W., M. Roginsky, and H. F. Moo. 1976. Abnormal vitamin D metabolism in patients with cirrhosis. Dig. Dis. 21:527-532.

2. Lund, B., O. H. Sorensen, M. Hilden, and B. Lund. 1977. The 
hepatic conversion of vitamin $\mathrm{D}$ in alcoholics with varying degrees of liver affection. Acta Med. Scand. 202:221-224.

3. Long, R. G., M. R. Wills, R. K. Skinner, and S. Sherlock. 1976. Serum 25-hydroxyvitamin $\mathrm{D}$ in untreated parenchymal and cholestatic liver disease. Lancet. ii:650-652.

4. Horsting, M., and H. F. DeLuca. 1969. In vitro production of 25hydroxycholecalciferol. Biochem. Biophys. Res. Commun. 36:251-256.

5. Reed, J. S., S. C. Meredith, B. A. Nemchausky, I. H. Rosenberg, and J. L. Boyer. 1980. Bone disease in primary biliary cirrhosis: reversal of osteomalacia with oral 25-hydroxyvitamin D. Gastroenterology. 78: 512-517.

6. Long, R. G., E. Meinhard, R. T. Skinner, Z. Varghese, M. R. Wills, and S. Sherlock. 1978. Clinical, biochemical, and histological studies of osteomalacia, osteoporosis, and parathyroid function in chronic liver disease. Gut. 19:85-90.

7. Long, R. G. 1980. Hepatic osteodystrophy: outlook good but some problems unsolved. Gastroenterology. 78:644-647.

8. Paterson, C. R., and M. S. Losowsky. 1967. The bones in chronic liver disease. Scand. J. Gastroenterol. 2:293-300.

9. Herlong, H. F., R. R. Recker, and W. C. Maddrey. 1982. Bone disease in primary biliary cirrhosis: histologic features and response to 25-hydroxyvitamin D. Gastroenterology. 83:103-108.

10. Matloff, D. S., M. M. Kaplan, R. M. Neer, M. J. Goldberg, W. Bitman, and H. J. Wolfe. 1982. Osteoporosis in primary biliary cirrhosis: effects of 25-hydroxyvitamin $\mathrm{D}_{3}$ treatment. Gastroenterology. 83:97-102.

11. Mobarhan, S. A., R. M. Russell, R. R. Recker, D. B. Posner, F. L. Iber, and P. Miller. 1984. Metabolic bone disease in alcoholic cirrhosis: a comparison of the effect of vitamin $D_{2}, 25$-hydroxyvitamin $D_{3}$, or supportive treatment. Hepatology. 4:266-273.

12. Bengoa, J. M., M. D. Sitrin, S. Meredith, S. E. Kelly, N. Shah, A. L. Baker, and I. H. Rosenberg. 1984. Intestinal calcium absorption and vitamin D status in chronic cholestatic liver disease. Hepatology. 4: 261-265.

13. Posner, D. B., R. M. Russell, S. Absood, T. B. Connor, C. Davis, L. Martin, J. B. Williams, A. H. Norris, and C. Merchant. 1978. Effective 25-hydroxylation of vitamin $\mathrm{D}_{2}$ in alcoholic cirrhosis. Gastroenterology. 74:866-870.

14. Bouillon, R., P. VanKerkhove, and P. DeMoor. 1976. Characteristics of the vitamin D binding protein in different species. Calcif. Tissue Res. 21(suppl.):172-176.

15. Haddad, J. G. Jr. 1979. Transport of vitamin D metabolites. Clin. Ortho. Rel. Res. 142:249-261.
16. Silver, J., and M. Fainaru. 1979. Transport of vitamin D sterols in human plasma: effect of excess vitamin $D, 25$-hydroxyvitamin $D$, and 1,25-dihydroxyvitamin D. Eur. J. Clin. Invest. 9:433-438.

17. Bikle, D. D., P. K. Siiteri, E. Ryzen, J. G. Haddad, and E. Gee. 1985. Serum protein binding of 1,25-dihydroxyvitamin $D$ : a reevaluation by direct measurement of free metabolite levels. J. Clin. Endocrinol. Metab. 61:969-975.

18. Haddad, J. G., D. P. Aden, and M. A. Kowalski. 1983. Characterization of the human plasma binding protein for vitamin $D$ and its metabolites synthesized by the human hepatoma derived cell line Hep3B. J. Biol. Chem. 258:6850-6854.

19. Barragry, J. M., D. Corless, J. Auton, N. D. Carter, R. G. Long, J. D. Maxwell, and S. Switala. 1978. Plasma vitamin D-binding globulin in vitamin D deficiency and chronic liver disease. Clin. Chim. Acta. 87: 359-366.

20. Bikle, D. D., E. Gee, B. Halloran, and J. G. Haddad. 1984. Free 1,25-dihydroxyvitamin $D$ levels in serum from normal subjects, pregnant subjects, and subjects with liver disease. J. Clin. Invest. 74:1966-1971.

21. Hammond, G. L., J. A. Nisker, L. A. Jones, and P. K. Siiteri. 1980. Estimation of the percentage of free steroid in undiluted serum by centrifugal ultrafiltration dialysis. J. Biol. Chem. 255:5023-5026.

22. Eisman, J. A., R. M. Shepard, and H. F. DeLuca. 1977. Determination of 25-hydroxyvitamin $\mathrm{D}_{2}$ and 25-hydroxyvitamin $\mathrm{D}_{3}$ in human plasma using high pressure liquid chromatography. Anal. Biochem. 80: 298-305.

23. Walsh, P. G., and J. G. Haddad. 1982. "Rocket" immunoelectrophoresis assay of vitamin D-binding protein (GC globulin) in human serum. Clin. Chem. 28:1781-1783.

24. Sitrin, M., S. Meredith, and I. H. Rosenberg. 1978. Vitamin D deficiency and bone disease in gastrointestinal disorders. Arch. Intern. Med. 138:886-888.

25. Wagonfeld, J. B., M. Bolt, J. L. Boyer, B. A. Nemchausky, J. V. Horst, and I. H. Rosenberg. 1976. Comparison of vitamin D and 25. hydroxyvitamin $\mathrm{D}$ in the therapy of primary biliary cirrhosis. Lancet. ii: 391-393.

26. Bikle, D. D., H. K. Genant, C. Cann, R. R. Recker, B. P. Halloran, and G. J. Strewler. 1985. Bone disease in alcohol abuse. Ann. Intern. Med. 103:42-48.

27. Bikle, D. D., E. Gee, B. Halloran, M. A. Kowalski, E. Ryzen, and J. G. Haddad. 1986. Assessment of the free fraction of 25-hydroxyvitamin $\mathrm{D}$ in serum and its regulation by albumin and vitamin D-binding protein. J. Clin. Endocrinol. Metab. In press. 